standardised interview. Gender, age, residence area and smoking were collected as potential confounders. Multivariate logistic regression was applied.

Results Considering all tumours together, we observed large increased risks for wood exposure $(\mathrm{OR}=6.9,95 \% \mathrm{CI}=3.0-16.3)$ and leather (prevalence $24 \%$ in tumours, $0 \%$ among controls) only. Compared to controls, we observed an increased risk for wood exposure $[\mathrm{OR}=7.7(95 \% \mathrm{CI}=2.6-22.5)]$ in ITAC cases, but not in non-ITAC cases $[\mathrm{OR}=0.8(95 \% \mathrm{CI}=0.2-3.1)]$. Prevalence of leather exposure was $42 \%$ among ITAC and $6 \%$ in non-ITAC.

Conclusions Our case control study confirmed that ITAC cases but not other histotypes were strongly related to occupational exposures, and in particular to leather and wood dusts. Grouping together all SNC types reduce the causal role of occupation exposures. Larger samples size are needed to investigate other work-related carcinogens.

\section{DO FAMILY AND INDIVIDUAL CHARACTERISTICS AFFECT THE EXPERIENCE OF PHYSICAL AND PSYCHOSOCIAL WORK ENVIRONMENT IN DANISH 20/21 YEAR OLDS?}

${ }^{1}$ Trine Nohr Winding, ${ }^{1,2}$ Merete Labriola, ${ }^{3,4}{ }^{2}$ Ellen Aagaard Nohr, ${ }^{1}$ Johan Hviid Andersen. ${ }^{1}$ Danish Ramazzini Centre, Department of Occupational Medicine, Regional Hospital Herning, Herning, Denmark; ${ }^{2}$ Department of Clinical Social Medicine, Public Health and Quality Management, Central Denmark Region and Section of Clinical Social Med, and Rehabilitation, School of Public Health, Aarhus University, Aarhus, Denmark; ${ }^{3}$ University of Southern Denmark, Institute of Clinical Research, Research Unit of Gynaecology and Obstetrics, Odense, Denmark; ${ }^{4}$ Department of Public Health, Section for Epidemiology, Aarhus University, Aarhus, Denmark

\subsection{6/oemed-2014-102362.68}

Objectives To describe the work environment of Danish 20/21 year olds and to investigate the influence of family socioeconomic background or individual characteristics at age $14 / 15$ on later experience of physical and psychosocial work environment.

Method The study population consisted of 695 young people with primary work affiliation at age $20 / 21$ who were derived from a prospective youth cohort. Outcome information from the questionnaire in 2010 consisted of six questions about psychosocial work environment and two questions about physical work environment. Exposure information about school performance, vulnerability, health and parental socioeconomic status was derived from the questionnaire in 2004 and from registers.

Results Overall, the psychosocial work environment of the young people was good but they experienced more repetitive movements and hard physical work than older workers. Individual as well as family factors in late childhood all together only had limited impact on how young people report later work environment. Low self-esteem at age 14/15 was associated with experiencing high demands, low trust and low fairness at work. In girls low self-esteem and low sense of meaningfulness were associated with experiencing low influence at work. Low parental socioeconomic status was associated with poor physical work environment.

Conclusions This study showed a social gradient in experiencing poor physical work environment at age 20/21. The psychosocial work environment in young people was on average good, but it seems that vulnerable young people need special intention in order to prevent them from being selected into psychosocial demanding job functions later in life.

\section{3}

GROUPING STRATEGIES FOR EXPOSURE ASSESSMENT OF THE PSYCHOSOCIAL WORK ENVIRONMENT

${ }^{1}$ Morten Vejs Willert, ${ }^{1,2}$ Vivi Schlünssen, ${ }^{2}$ loannis Basinas, ${ }^{1}$ Zara Ann Stokholm, ${ }^{3}$ Matias Brødsgaard Grynderup, ${ }^{4}$ Johan Hviid Andersen, ${ }^{5}$ Reiner Rugulies, ${ }^{3}$ Åse Marie Hansen, ${ }^{6}$ Linda Kærlev, ${ }^{7}$ Jane Frolund Thomsen, ${ }^{7}$ Marianne Agergaard Vammen, ${ }^{1}$ Henrik Kolstad. ${ }^{1}$ Danish Ramazzini Centre, Department of Occupational Medicine, Aarhus University Hospital, Region Midt, Denmark; ${ }^{2}$ Danish Ramazzini Centre, Department of Occupational Medicine, Herning University Hospital, Region Midt, Denmark; ${ }^{3}$ Research Unit of Clinical Epidemiology, Institute of Clinical Research, University of Southern Denmark, Region Southern Denmark, Denmark; ${ }^{4}$ Department of Public Health, Aarhus University, Aarhus, Denmark; ${ }^{5}$ Department of Public Health, University of Copenhagen, Copenhagen, Denmark; ${ }^{6}$ Research Centre for the Working Environment, Copenhagen, Denmark; 'Department of Occupational and Environmental Medicine, Bispebjerg University Hospital, Capital Region, Denmark

\subsection{6/oemed-2014-102362.69}

Objectives Individual response style, mood, expectations, and health status may affect reporting of the psychosocial work environment, and bias associations with outcomes. Reporting bias may be avoided by aggregating individual responses, ideally preserving exposure contrast. In this study, we examined the degree of exposure contrast yielded by different grouping strategies. Method In 2007, we enrolled 4489 public employees from Aarhus, Denmark in the PRISME-cohort, with follow-up in 2009. From pay-roll registers we grouped workers at 2 organisational levels: department $(\mathrm{n}=22)$ and work unit $(\mathrm{n}=751)$, and 3 occupational levels: sector $(n=7)$, profession $(n=46)$, and job title $(\mathrm{n}=77)$. Exposures, calculated as means of items scored on 5 -point Likert scales, included psychological demands, decision latitude, social support, effort, reward, and procedural and relational justice. To assess variance components, we fitted linear mixed effect models with exposures as dependent variables, and id and grouping variables as random effects. Results are reported as the contrast in mean exposure levels e.g. between-group variance/ (between-group variance + within-group variance).

Results Within each hierarchy contrasts rose with increasing grouplevel detail. Grouping by either work unit (wu) or by job title (jt) contrasts were: psychological demands: $0.28(\mathrm{wu}) ; 0.26(\mathrm{jt})$, decision latitude: $0.24(\mathrm{wu}) ; 0.32(\mathrm{jt})$, social support: $0.24(\mathrm{wu}) ; 0.06(\mathrm{jt})$, effort: $0.23(\mathrm{wu}) ; 0.16(\mathrm{jt})$, reward: $0.19(\mathrm{wu}) ; 0.12(\mathrm{jt})$, procedural justice: $0.24(\mathrm{wu}) ; 0.14(\mathrm{jt})$, and relational justice: $0.29(\mathrm{wu}) ; 0.04(\mathrm{jt})$. Conclusions Grouping by work unit gave the most consistent contrasts $(0.19-0.29)$, while grouping by job title varied considerably (0.04-0.32). These preliminary findings suggest that grouping by work unit provided better exposure contrasts than grouping by job title for all exposures, but decision latitude.

\section{PREDICTORS OF WORKERS' COMPENSATION CLAIM DURATION AMONG WORKERS DISABLED DUE TO LOW BACK PAIN}

${ }^{1}$ Jason Busse, ${ }^{2}$ Ivan Steenstra, 'Shanil Ebrahim, 'Diane Heels-Ansdell, 'Stephen Walter, ${ }^{1}$ Gordon Guyatt. ${ }^{1}$ McMaster University, Hamilton, Ontario, Canada, ${ }^{2}$ Institute for Work and Health, Toronto, Ontario, Canada

\subsection{6/oemed-2014-102362.70}

Objectives Low back pain (LBP) is a common complaint among workers receiving Workers' Compensation wage replacement benefits. We used the administrative data from the Ontario Workplace Safety and Insurance Board (WSIB) to explore the association between baseline characteristics and commonly reimbursed therapies and time to claim closure among workers disabled due to LBP. 
Method Using the WSIB administrative database, we acquired a random sample of 6665 injured workers who reported an uncomplicated back injury (strain or sprain) with a date of injury between January 1, 2005 and June 30, 2005. We selected, a priori, 11 variables from the database that we judged may be associated with claim closure and predicted the direction of anticipated effects. We performed a time-to-event analysis using Cox proportional hazards regression to assess the association between time to claim closure and the independent variables. Receipt of WSIB-reimbursed chiropractic care or physiotherapy were treated as a time-dependent covariate to account for when treatment was initiated during the course of the disability claim.

Results Our adjusted regression analysis showed that older age and opioid prescription (adjusted hazard ratio $[\mathrm{HR}]=0.69 ; 99 \%$ $\mathrm{CI}=0.53,0.89)$ in the first 4 -weeks of claim reimbursed by the WSIB were associated with prolonged claim closure, whereas working for an employer that had a return-to-work program was associated with shorter claim duration (adjusted $\mathrm{HR}=1.73$; 99\% CI=1.42, 2.12). Neither reimbursement for chiropractic or physiotherapy were associated time to claim closure.

Conclusions Our analysis suggests that commonly reimbursed treatment for Workers' Compensation LBP claimants may be ineffective or even harmful.

\section{FACE VALIDITY AND INTER-RATER AGREEMENT BETWEEN EXPERT ASSESSMENTS OF OCCUPATIONAL MECHANICAL EXPOSURES IN A LOWER BODY JOB EXPOSURE MATRIX}

${ }^{1}$ Poul Frost, ${ }^{2}$ Tine Steen Rubak, ${ }^{3}$ Johan Hviid Andersen, ${ }^{3}$ Jens Peder Haahr ${ }^{4}$ Ann Isabel Kryger, ${ }^{1}$ Lone Donbæk Jensen, ${ }^{3}$ Susanne Wulff Svendsen. 'Danish Ramazzini Centre, Department of Occupational Medicine, Aarhus University Hospital, Aarhus, Denmark; '2Deparment of Occupational Medicine, Slagelse Hospital, Slagelse, Denmark; ${ }^{3}$ Danish Ramazzini Centre, University Department of Occupational Medicine, Herning Regional Hospital, Herning, Denmark; ${ }^{4}$ Department of Occupational and Environmental Medicine, Bispebjerg Hospital, Copenhagen, Denmark

\subsection{6/oemed-2014-102362.71}

Objectives We constructed a lower body job exposure matrix (JEM) based on five experts' assessments of occupational mechanical exposures. The aim of this study was to evaluate the face validity of the rankings of the job groups and the inter-rater agreement between the experts' rankings.

Method The JEM cross tabulates the mean of five experts' assessments of daily duration (hours/day) of standing/walking, kneeling/squatting, and whole-body vibration as well as total load lifted ( $\mathrm{kg} /$ day), and frequency of lifting loads weighing $\geq 20$ $\mathrm{kg}$ (times/day) in 121 job groups comprising occupational titles with expected homogeneous exposure patterns. The JEM covers 689 occupational titles, which were considered more than minimally exposed, out of 2227 in the Danish version of the International Standard Classification of Occupations. Weighted kappa statistics were used to evaluate inter-rater agreement on rankings of the job groups for four of these exposures. Two external experts checked the face validity of the rankings of the experts' mean values.

Results The experts' ratings showed fair to moderate agreement (mean weighted kappa values between 0.36 and 0.49 ). The external experts agreed on 586 of the 605 rankings.

Conclusions Experts agreed on rankings of the job groups, and rankings based on mean values had good face validity. However, further validation is warranted based on technical measurements or observations. The lower body JEM, which provides exposure estimates free of recall bias, has been applicable in exposure response studies of hip and knee osteoarthritis, inguinal hernia repair, varicose veins, and lumbar disc disorders.

\section{MORTALITY PROFILE OF THE FRENCH COHORT OF URANIUM PROCESSING WORKERS}

${ }^{1}$ Eric Samson, ${ }^{1}$ Irwin Piot, ${ }^{1}$ Sergey Zhivin, ${ }^{2}$ Alain Acker, ${ }^{2}$ Pierre Laroche, ${ }^{1}$ Dominique Laurier. ${ }^{1}$ IRSN, Fontenay-Aux-Roses, France, ${ }^{2}$ AREVA, Paris, France

\subsection{6/oemed-2014-102362.72}

Objectives A cohort of nuclear workers employed in the fuel processing cycle was set-up in France in 2009 to assess the risk of cancer and non-cancer mortality related to uranium, in a context of occupational multi-exposure. Vital status and causes of death are now available.

Method The cohort includes workers employed at least 6 months between 1958 and 2006 by AREVA and CEA (French Atomic Energy Commission). Exposure assessment was realised by a dual approach combining individual monitoring data and specific job-exposure matrices, considering radiation exposures, chemical agents and physical stresses (heat and noise). Additional information like tobacco consumption and various clinical parameters (Body Mass Index, blood pressure, lipid profile etc.) was also collected from the Occupational Health Services. Vital status and causes of death were collected from French national registries.

Results The cohort includes 12739 workers (88\% men) with an average duration of employment of 17 years. The median year of birth is 1944. At the end of follow-up (31/12/2010), 19\% are deceased and $96 \%$ of the causes of death are identified. Cancers (mainly lung, prostate and lymphatic and haematopoietic tissue cancers) represent $43 \%$ of all causes of death, non-cancers (mainly diseases of the circulatory system) $48 \%$ and external causes 9\%. The analyses using French national mortality rates as reference will be presented.

Conclusions The observed mortality is that of an even young population and at this stage no further conclusions can be drawn. This cohort, with this wealth of data, will be very informative for the investigation of uranium related risks.

\section{ALCOHOL CONSUMPTION AS A CONFOUNDER IN NEUROBEHAVIORAL STUDIES OF NEUROTOXICANTS}

${ }^{1}$ Rita Bast-Pettersen, ${ }^{2}$ Maxim Chashchin, ${ }^{2}$ Valery Chashchin, ${ }^{1}$ Yngvar Thomassen, ${ }^{1}$ Dag G Ellingsen. ' National Institute of Occupational Health, Os/o, Norway; ${ }^{2}$ Northwest Public Health Research Centre, St. Petersburg, Russia

\subsection{6/oemed-2014-102362.73}

Objectives To study the effects of manganese (Mn) exposure and alcohol consumption on tremor.

Method Tremor was measured in 137 shipyard welders (age 39.9) and 137 referents (turner/fitters) (age 40.1) with the Catsys TREMOR. Alcohol consumption was assessed by measuring serum carbohydrate deficient transferrin (sCDT).

Results The geometric mean (GM) of $\mathrm{Mn}$ air concentration was $214 \mu \mathrm{g} / \mathrm{m}^{3}$ (range 1-3230). The GM concentrations of $\mathrm{Mn}$ in whole blood (B-Mn) and urine (U-Mn) were $12.8 \mu \mathrm{g} / \mathrm{L}$ and $0.36 \mu \mathrm{g} / \mathrm{g}$ creatinine versus $8.0 \mu \mathrm{g} / \mathrm{L}$ and $0.07 \mu \mathrm{g} / \mathrm{g}$ creatinine in the referents. Concentration of sCDT (\%) was 0.71 in welders and 0.65 in referents. 